\title{
Management of Patients with COVID-19 Infection in Acute Settings: A Position Paper for Cardiopulmonary Physiotherapists by the Association of Clinical and Academic Physiotherapists of Nigeria
}

\author{
Rufus A. Adedoyin ${ }^{1}$, Jibril Mohammed ${ }^{2}$, Chidiebele P. Ojukwu ${ }^{3}$, Udoka A. C. Okafor ${ }^{4}$, \\ Ifeanyi K. Oti ${ }^{5}$, Chioma L. Onuchukwu ${ }^{6}$, Samuel O. Famude ${ }^{7}$, Emmanuel S. Aliyu, \\ Ifeoma B. Nwosu' ${ }^{9}$, Ernest E. Anikwe ${ }^{10}$, Adesola C. Odole ${ }^{11}$, Rita N. Ativie ${ }^{3}$, \\ Nse A. Odunaiya11, Happiness A. Aweto ${ }^{4}$, Caleb A. O. Gbiri ${ }^{4}$ \\ ${ }^{1}$ Department of Medical Rehabilitation, Obafemi Awolowo University, Ile-Ife, Nigeria \\ ${ }^{2}$ Physiotherapy Department, Bayero University, Kano, Nigeria \\ ${ }^{3}$ Department of Medical Rehabilitation, University of Nigeria, Enugu Campus, Enugu, Nigeria \\ ${ }^{4}$ Physiotherapy Department, University of Lagos, Lagos, Nigeria \\ ${ }^{5}$ Physiotherapy Department, University Teaching Hospital, Port-harcout, Nigeria \\ ${ }^{6}$ Physiotherapy Department, Enugu State University Teaching Hospital, Enugu, Nigeria \\ ${ }^{7}$ Physiotherapy Department, Lagos State University Teaching Hospital, Ikeja, Lagos, Nigeria \\ ${ }^{8}$ Physiotherapy Department, University of Abuja Teaching Hospital, Gwagwalada, Abuja \\ ${ }^{9}$ Department of Medical Rehabilitation, Nnamdi Azikwe University, Nnewi, Akwa, Campus, Nigeria \\ ${ }^{10}$ Physiotherapy Department, University College Hospital, Ibadan, Nigeria \\ ${ }^{11}$ Physiotherapy Department, University of Ibadan, Ibadan, Nigeria \\ Email: *radedoyi@yahoo.com
}

How to cite this paper: Adedoyin, R.A., Mohammed, J., Ojukwu, C.P., Okafor, U.A.C., Oti, I.K., Onuchukwu, C.L., Famude, S.O., Aliyu, E.S., Nwosu, I.B., Anikwe, E.E., Odole, A.C., Ativie, R.N., Odunaiya, N.A., Aweto, H.A. and Gbiri, C.A.O. (2020) Management of Patients with COVID-19 Infection in Acute Settings: A Position Paper for Cardiopulmonary Physiotherapists by the Association of Clinical and Academic Physiotherapists of Nigeria. Open Journal of Therapy and Rehabilitation, 8, 72-82. https://doi.org/10.4236/ojtr.2020.83007

Received: May 11, 2020

Accepted: August 2, 2020

Published: August 5, 2020

\begin{abstract}
Coronavirus Disease-2019 (COVID-19) refers to a clinical disorder caused by a new coronavirus that emerged in late 2019. According to the European Centre for Disease Prevention and Control, as of June 23 2020, over 15 million confirmed cases of patients with COVID-19 have been reported globally, out of which over 600,000 deaths were recorded. The management of patients with COVID-19 is critical. This position paper was prepared through online search for the available guideline in the literature to serve as treatment guidelines for Nigerian physiotherapists and possibly physiotherapists in Sub-Saharan Africa region.
\end{abstract}

\section{Keywords}

COVID-19, Respiratory Disease, Prevention, Physiotherapy, Nigeria 
Copyright $\odot 2020$ by author(s) and Scientific Research Publishing Inc. This work is licensed under the Creative Commons Attribution International License (CC BY 4.0).

http://creativecommons.org/licenses/by/4.0/

\section{Introduction}

Coronavirus Disease-2019 (COVID-19) refers to a clinical disorder caused by a new coronavirus that emerged in late 2019.The history of COVID-19 is traced to an outbreak of a mysterious pneumonia that is characterized by clinical features of fever, dry cough, dyspnoea, and fatigue, and occasional gastrointestinal symptoms, with its first observation in Huanan Seafood Wholesale Market, in Wuhan, Hubei, China in late December 2019 [1]. Subsequently, thousands of people in China, especially in the Wuhan region, were infected by the rampant spread of the corona virus [2]. The COVID-19 has consistently spread to several countries of the world, resulting in serious collapse of health care systems and services as well as significant morbidities and mortalities. It was thus considered a Public Health Emergency of international concern and a pandemic [3]. According to the European Centre for Disease Prevention and Control, as of 2020, over 15 million confirmed cases of patients with COVID-19 have been reported globally, out of which $600,000+$ deaths were recorded [4]. So far, Africa has recorded over 16,430 deaths from the disease with most deaths occurring in South Africa (5940), Egypt (4440), Algeria (1111), Nigeria (813) and Sudan (706) [4].

The Nigeria Centre for Disease Control (NCDC) reported that as at July 24 2020 , over $251,000+$ individuals have been tested among which $38,000+$ cases were confirmed to be positive. Out of these, 16,000+ have been successfully managed and discharged while 833 deaths have been recorded [5]. It is important to note that ever since COVID-19 was first detected in Nigeria, there has been a steady increase in the prevalence of the disease, which has necessitated shutting down many cities and states in the country. Other measures taken by the Nigerian government included suspension of international and local air travel to and from the country, banning of inter-state travels across several states, and directing citizens (with the exception of essential service providers) have been directed to "stay-at-home" (with the exception of essential service providers) or possibly work from home, as measures to combat the spread of the disease [6].

Nevertheless, several analysts have expressed concern about the "true" state of the COVID-19 data presented by the NCDC in Nigeria in view of the limited testing capacity across the country. Moreover, only a small fraction of the population has been tested. States such as Kano in recent times have recorded unprecedented surge in deaths of individuals fitting the mortality pattern from COVID-19 [7]. Furthermore, widespread community transmission of the disease has been suspected even before the Nigerian Government declared lockdown and movement restrictions. Therefore, there is a high possibility that COVID-19 is underdiagnosed and underestimated in Nigeria.

Susceptibility to COVID-19 is associated with age, sex and other health conditions or comorbidities [8]. COVID-19 reported to be prevalent among the elderly and male sex. Despite the fact that most infected individuals may be asymptomatic, symptoms experienced by patients with COVID-19 who are indicated 
for treatment span across multiple systems; respiratory (cough, short of breath, sore throat, rhinorrhoea, haemoptysis, and chest pain), gastrointestinal (diarrhoea, nausea, and vomiting), musculoskeletal (muscle ache), and neurologic (headache or confusion) [8]. Of note, patients affected by COVID-19 who need hospitalisation often present with viral pneumonia, that is often complicated by an acute respiratory failure, which may eventually evolve to acute respiratory distress syndrome [9].

Considering that pneumonia and Acute Respiratory Distress Syndrome (ARDS) are the commonest associated complications of COVID-19. There may be a resultant alteration of the ventilation-perfusion ratio with possible shunt [10]. Moreover, acute hypoxemic patients may experience dyspnoea which may persist despite the administration of oxygen flows (of $>10-15 \mathrm{~L} / \mathrm{min}$ ) using a reservoir mask [11]. However, some devices, such as High-Flow Nasal Oxygen (HFNO), Continuous Positive Airways Pressure (CPAP) or Non-Invasive Ventilation (NIV) have been found to be useful [12].

In the presence of acute respiratory failure, there is a reduction of lung compliance, increased respiratory work of breathing and alteration of blood oxygenation, leading to a rapid and shallow respiratory pattern [13]. Usually, this pattern is spontaneously adopted by the subject as a strategy to minimize inspiratory effort and maximize mechanical efficiency of breathing. Additionally, in such clinical conditions, the strength of the respiratory muscles can be reduced. It is important that treatments and procedures used by physiotherapists do not cause a further burden on the work of breathing, exposing the patient to an increased risk of respiratory distress. Consequently, experts have cautioned against the use of diaphragmatic breathing-pursed lips breathing-bronchial hygiene/lung re-expansion techniques (PEP Bottle, EzPAP ${ }^{\circledR}$, cough machines, incentive spirometer-manual mobilization/stretching of the rib cage in the presence of acute respiratory failure that determines a reduction of lung compliance, because the increase in the respiratory work of breathing and alteration of blood oxygenation may lead to rapid and shallow respiratory pattern, while treating patients with COVID-19 [14].

Some form of respiratory or general physiotherapy may be indicated in such patients on a case by case basis. Cardiopulmonary physiotherapists are involved in the management and care of patients with COVID-19 in the acute hospital setting, and play a key role in non-invasive support management, postural changes, mobilisation and during weaning from invasive mechanical ventilator support. Physiotherapists are also involved in the rehabilitation period following recovery from the disease. A number of reputable international organisations such as the World Confederation of Physical Therapists and the European Respiratory Society have published international guidelines for physiotherapy management of COVID-19 on their websites. Most of the published guidelines have been either adopted, adapted or endorsed by national or specialized associations, notably: Australian Physiotherapy Association, Canadian Physiotherapy Association, In- 
ternational Confederation of Cardiorespiratory Physical Therapists, Italian Association of Respiratory Physiotherapists [9] [15].

In view of the rising cases of patients with COVID-19 in Nigeria, there is a very high possibility, in the nearest future, for the need for more specialized care such as respiratory physiotherapy, to manage patients with indications for physiotherapy. Moreover, most hospitals in Nigeria have limited capacity in terms of number of bed spaces, especially in the intensive care units (ICU). There is already severe shortage of personal protective equipment (gears) for use by healthcare practitioners. It is also possible that physiotherapists could be exposed to other asymptomatic patients who may not have been tested for COVID-19, but potentially infectious. Therefore, this document is prepared to provide guidelines for Nigerian physiotherapists who may be called upon in the management of patients with confirmed COVID-19.

\section{Overview of Assessment}

Relevant relevant assessment should be done by a respiratory physiotherapist prior to treatment as indicated in the subject headings below:

\section{Signs of respiratory abnormalities}

A respiratory assessment is an external assessment of ventilation that includes observations of the rate, depth and pattern of respirations. While assessing for signs and symptoms of respiratory changes, emphasis should be placed on the following:

1) Complaints of shortness of breath (dyspnea);

2) Bluish or cyanotic appearance of the nail beds lips, mucous membranes;

3) Restlessness, irritability, confusion, decreased level of consciousness;

4) Pain on inspiration and expiration;

5) Laboured or difficult breathing;

6) Orthopnea;

7) Use of accessory respiratory muscles;

8) Abnormal breath sounds such as wheezes, rhonchi or rales;

9) Inability to breathe spontaneously;

10) Thick, frothy, blood-tinged or copious sputum production;

11) Paradoxical chest wall movement.

\section{Assessment of breath sounds}

Normal breath sounds or vesicular sound: these are low-pitched, soft blowing sounds heard throughout the lung fields that occur throughout inspiration.

Abnormal breath sounds: 1) tracheal-high-pitched, harsh tubular sounds heard over the trachea and throat; 2) bronchial-high-pitched tubular sounds over the large airways of the chest; 3 ) bronchovesicular-tubular sounds, best heard posteriorly between the scapulae; 4) crackles-popping or bubbling sounds that occur when air is forced through fluid-filled airways; and 5) plural-fiction rub: A cracking sound caused by the moves quickly through mucus-filled, narrowed airways [16] [17] [18] [19] [20]. 


\section{Significance of This Paper}

The recommendation in this paper follows international standards as they were drawn from web search and international guidelines for COVID-19 management of critically ill patients with COVID-19. (Thomas et al. [21]; World Health Organization, professional bodies such as Italian Association of Respiratory Physiotherapists, World Confederation of Physical Therapy). The guidelines were modified to conform with the specific peculiarity of the Nigerian context. The initial draft was made by experts in the field of cardiopulmonary physiotherapy and was later reviewed by senior Physiotherapy academics. Thomas et al. [21] previously presented a list in a comprehensive document for use by physiotherapists in the management of COVID-19. This list has been adapted to the Nigerian context and presented in Tables 1-5 below.

Table 1. Physiotherapy workforce planning and preparation recommendations.

1.1 Increment in the number of cardiopulmonary trained physiotherapists.

1.2 Recruitment of more physiotherapists into the work force by the Ministry of Health, Nigeria.

1.3 Recall those on leave back to work.

1.4 Involvement of University academics into clinical settings.

1.5 Recall retired staff with previous experiences in cardiorespiratory and critical care.

1.6 Extend retirement age of clinical staff.

1.7 Physiotherapists should have specialised knowledge, skills and decision-making to work within the ICU. Physiotherapists with previous ICU experience should be requested to work.

1.8 Physiotherapists who do not have recent cardiorespiratory physiotherapy experience should be urgently trained to offer services to cope with the management of patients with COVID-19.

1.9 Staff with advanced ICU physiotherapy skills should be supported to screen patients with COVID-19 assigned to physiotherapy caseloads and provide junior ICU staff with appropriate supervision and support, particularly with decision-making for complex patients with COVID-19.

2.0 Identify existing learning resources for staff who could be deployed to ICU. For example:

- e-learning packages (e.g., Clinical Skills Development Service for Physiotherapy and Critical Care Management)

- Local Physiotherapy Staff ICU Orientation

- PPE training

2.1 Keep staff informed of plans. Communication is crucial to the successful delivery of safe and effective clinical services.

2.2 Staff who are judged to be at high risk should be exempted from COVID-19 isolation areas.

When planning staffing and rosters, the following people may be at higher risk of developing more serious illness from COVIDD-19 and should avoid exposure to patients with COVID-19. This includes staff who: are pregnant, have significant chronic respiratory illnesses, are immune-suppressed, older (e.g., $>60$ years), have severe chronic health conditions such as heart disease, lung disease, diabetes, immune deficiencies, such as neutropenia, disseminated malignancy and conditions or treatments.

Workforce planning should include consideration for pandemic-specific requirements such as additional workload from donning and doffing PPE, and the need to allocate staff to key non-clinical duties such as enforcing infection control procedures.

Reorganising the workforce into teams that will manage COVID-19 versus non-infectious patients. Minimise or prevent movement of staff between teams. Liaise with local infection control services for recommendations. 


\section{Continued}

2.3 Be aware of and comply with relevant international, National, state and/or hospital guidelines for infection control in healthcare facilities. For example, World Health Organization "Guidelines for infection prevention and control during health care when novel coronavirus infection is suspected".

2.4 Senior physiotherapists should be involved in determining the appropriateness of physiotherapy interventions for patients with suspected and/or proven COVID-19 in consultation with senior medical staff and according to a referral guideline.

2.5 Identify additional physical resources that may be required for physiotherapy interventions and how the risk of cross-infection can be minimised (e.g., respiratory equipment; mobilisation, exercise and rehabilitation equipment; and equipment storage).

2.6 Identify and develop a facility inventory of respiratory, mobilisation, exercise and rehabilitation equipment and determine the process of equipment allocation as pandemic levels increase (i.e., to prevent movement of equipment between infectious and non-infectious areas).

2.7 It should be recognised that staff will likely have an increased workload with a heightened risk of anxiety both at work and home. Staff should be supported during and beyond the active treatment phases (e.g., via access to employee assistance programs, counselling and facilitated debriefing sessions).

2.8 Consider and/or promote debriefing and psychological support; staff morale may be adversely affected due to the increased workload, anxiety over personal safety and the health of family members.

Table 2. COVID-19 indications for physiotherapy.

2.1 The respiratory infection associated with COVID-19 is mostly associated with a dry and non-productive cough; lower respiratory tract involvement usually involves pneumonitis rather than exudative consolidation.In these cases, respiratory physiotherapy interventions are not indicated.

2.2 Respiratory physiotherapy interventions in hospital wards or ICU may be indicated for patients who have suspected or confirmed COVID-19 and concurrently or subsequently develop exudative consolidation, mucous hypersecretion and/or difficulty clearing secretions.

2.3 Physiotherapists are responsible for providing interventions for mobilisation, exercise and rehabilitation (e.g. in patients with comorbidities creating significant functional decline and/or (at risk) for ICU-acquired weakness).

2.4 Physiotherapy interventions should only be provided when there are clinical indicators, so that staff exposure to patients with COVID-19 is minimised. Unnecessary review of patients with COVID-19 within their isolation room/areas will also have a negative impact on PPE supplies.

2.5 Physiotherapists should meet regularly with senior medical staff to determine indications for physiotherapy review in patients with confirmed or suspected CoViD-19 and screen according to set/agreed guidelines.

2.6. Physiotherapy staff should not be routinely entering isolation rooms, where patients with confirmed or suspected COVID-19 are isolated.

2.7 Options for screening patients via subjective review and basic assessment whilst not being in direct contact with the patient should be trialled first whenever possible (e.g., calling the patient's isolation room telephone and conducting a subjective assessment for mobility information and/or providing education on airway clearance techniques).

Table 3. Recommendations for physiotherapy respiratory interventions.

3.1 It is strongly recommended that airborne precautions are utilised during respiratory physiotherapy interventions.

\section{Cough etiquette}

3.2 Both patients and staff should practise cough etiquette and hygiene.

During techniques that may provoke a cough, education should be provided to enhance cough etiquette and hygiene

- Ask patient to cover their mouth by coughing into their elbow or sleeve or into a tissue. Tissues should then be disposed and hand hygiene performed.

- In addition, if possible, physiotherapists should position themselves $\geq 2 \mathrm{~m}$ from the patient and out of the likely path of dispersion. 


\section{Continued}

\section{Aerosol-generating procedures}

3.3 Many respiratory physiotherapy interventions are potentially aerosol-generating procedures. While there are insufficient investigations confirming the aerosol generating potential of various physiotherapy interventions, the combination with cough for airway clearance makes all techniques potentially aerosol-generating procedures.

These include:

- cough-generating procedures (e.g. cough during treatment or huff)

- positioning or gravity-assisted drainage techniques and manual techniques (e.g., expiratory vibrations, percussion and manual assisted cough) that may trigger a cough and sputum expectoration

- use of positive pressure breathing devices (e.g. inspiratory positive pressure breathing), mechanical insufflation-exsufflation devices, intra/extra pulmonary high-frequency oscillation devices (e.g. The Vest, MetaNeb, Percussionaire)

- PEP and oscillating PEP devices

- Bubble PEP

- Nasopharyngeal or oropharyngeal suctioning

- Manual hyperinflation

- Open suction

- Saline instillation via an open-circuit endotracheal tube

- Inspiratory muscle training, particularly if used with patients who are ventilated and disconnection from a breathing circuit is required

- Sputum inductions

- Any mobilisation or therapy that may result in coughing and expectoration of mucus

Therefore, there is a risk of creating an airborne transmission of COVID-19 during treatments. Physiotherapists should weigh up the risk versus benefit to completing these interventions and use airborne precautions.

3.4 Where aerosol-generating procedures are indicated and considered essential, they should be undertaken in a negative-pressure room, if available, or in a single room with the door closed. Only the minimum number of required staff should be present and they must all wear PPE, as described. Entry and exit from the room should be minimised during the procedure.

This may not be able to be maintained when cohorting is required because of the volume of patients presenting with COVID-19.

3.5 Bubble PEP is not recommended for patients with COVID-19 because of uncertainty around the potential for aerosolisation, which is similar to the caution the WHO places on bubble CPAP.

3.6 There is no evidence for using incentive spirometry in patients with COVID-19.

3.7 Avoid the use of mechanical insufflation/exsufflation, non-invasive ventilation, inspiratory positive pressure breathing devices or high-flow nasal oxygen devices.

However, if clinically indicated and alternative options have been ineffective, consult with both senior medical staff and Infection Prevention and Monitoring Services within local facilities prior to its use.

If used, ensure that machines can be decontaminated after use and protect machines with viral filters over machine and patient ends of circuits.

- Use disposable circuits for these devices

- Maintain a log for devices that includes patient details for tracking and infection

- Monitoring (if required)

- Use airborne precautions'

3.8 Where respiratory equipment is used, whenever possible, use single-patient-use disposable options (e.g., single-patient-use PEP devices). Re-usable respiratory equipment should be avoided, where possible.

3.9 Physiotherapists should not implement humidification, non-invasive ventilation or other aerosol-generating procedures without consultation and agreement with a senior doctor (e.g, medical consultant).

\section{Sputum inductions}

3.10 Sputum inductions should not be performed.

\section{Requests for sputum samples}

3.11 In the first instance, ascertain whether the patient is productive of sputum and able to clear sputum independently. If so, physiotherapy is not required for a sputum sample. If physiotherapy interventions are required to facilitate a sputum sample, full airborne, full PPE should be worn. The handling of sputum samples should adhere to local policies. Generally, once a sputum sample has been obtained the following points should be followed:

- All sputum specimens and request forms should be marked with a biohazard label. 


\section{Continued}

- The specimen should be double-bagged. The specimen should be placed in the first bag in the isolation room by a staff member wearing recommended PPE.

Specimens should be hand-delivered to the laboratory by someone who understands the nature of the specimens. Pneumatic tube systems must not be used to transport specimens.

\section{Saline Nebulisation}

3.12 Do not use saline nebulisation.

\section{Manual hyperinflation}

3.13 As it involves disconnection/opening of a ventilator circuit, avoid manual hyperinflation and utilise ventilator hyperinflation if indicated (e.g., for suppurative presentations in ICU and if local procedures are in place).

\section{Positioning, including gravity-assisted drainage}

3.14 Physiotherapists can continue to advice on positioning requirements for patients.

\section{Prone Positioning}

3.15 Physiotherapists may have a role in the implementation of prone positioning in the ICU. This may include leadership within ICU 'prone teams', providing staff education on prone positioning (e.g., simulation-based education sessions) or assisting in turns as part of the ICU team.

\section{Tracheostomy Management}

3.16 The presence of a tracheostomy and related procedures that are potentially aerosol generating.

- Cuff deflation trials and inner tube changes/cleaning can be aerosol generating.

- Closed, in-line suction is recommended.

- Inspiratory muscle training, speaking valves and leak speech should not be attempted until patients are over the acute infection and the risk of transmission is reduced.

- Airborne precautions are recommended with infectious patients with COVID-19 with a tracheostomy.

Table 4. Recommendations for Physiotherapy Mobilisation, Exercise and Rehabilitation Interventions.

\section{Personal protective equipment}

4.1 Droplet precautions should be appropriate for the provision of mobilisation, exercise and rehabilitation in most circumstances. However, physiotherapists are likely to be in close contact with the patient (e.g., for mobilisation, exercise or rehabilitation interventions that require assistance). In these cases, consider use of a high filtration mask (e.g., P2/N95). Mobilisation and exercise may also result in the patient coughing or expectorating mucus, and there may be circuit disconnections with ventilated patients.

\section{Screening}

4.2 Physiotherapists will actively screen and/or accept referrals for mobilisation, exercise and rehabilitation.

When screening, discussion with physician or family is recommended before deciding to enter the patient's isolation room. For example, to try to minimise staff who come in to contact with patients with COVID-19, physiotherapists may screen to determine an appropriate aid to trial.

4.3 Direct physiotherapy interventions should only be considered when there are significant functional limitations, such as (risk for) ICU-acquired weakness, frailty, multiple comorbidities and advanced age.

\section{Early Mobilisation}

4.4 Early mobilisation is encouraged. Actively mobilise the patient early in the course of illness when safe to do so.

4.5 Patients should be encouraged to maintain function, as able within their rooms.

- Sit out of bed

- Perform simple exercises and activities of daily living

\section{Mobilisation and exercise prescription}

4.6 Mobilisation and exercise prescription should involve careful consideration of the patient's state (e.g., stable clinical presentation with stable respiratory and haemodynamic function). 


\section{Continued}

\section{Mobility and exercise equipment}

4.7 The use of equipment should be carefully considered and discussed with local infection monitoring and prevention service staff before being used with patients with COVID-19 to ensure that it can be properly decontaminated.

4.8 Use equipment that can be single patient use. For example, use elastic resistance bands rather than distributing hand weights.

4.9 Larger equipment (e.g., mobility aids, ergometers, chairs and tilt tables) must be easily decontaminated. Avoid use of specialised equipment, unless necessary, for basic functional tasks. For example, stretcher chairs or tilt tables may be deemed appropriate if they can be decontaminated with appropriate cleaning and are indicated for progression of sitting/standing.

4.10 When mobilisation, exercise or rehabilitation interventions are indicated:

- Plan well

- Identify/use the minimum number of staff required to safely perform the activity

- Ensure that all equipment is available and working before entering rooms

- Ensure that all equipment is appropriately cleaned or decontaminated

- If equipment needs to be shared among patients, clean and disinfect between each patient use

- Specific staff training for cleaning of equipment within isolation rooms may be required

- Whenever possible, prevent the movement of equipment between infectious and non-infectious areas

- Whenever possible, keep dedicated equipment within the isolation zones, but avoid storing extraneous equipment within the patient's room

4.11 When performing activities with ventilated patients or patients with a tracheostomy, ensure that airway security is considered and maintained (e.g., a dedicated airway person to prevent inadvertent disconnection of ventilator connections/tubing).

Table 5. Recommendations regarding personal protective equipment for physiotherapists.

5.1 All staff must be trained in correct donning and doffing of PPE, including N95 "fitchecking".

A registry of staff who have completed PPE education and fit checking should be maintained.

5.2 "Fit testing" is recommended when available, but the evidence for fit testing effectiveness is limited and the variation in supply of N95 mask types may make any recommendation on fit testing difficult to implement from a practical perspective.

5.3 Staff with beards should be encouraged to remove facial hair to ensure good mask fit.

5.4 For all suspected and confirmed cases, droplet precautions should be implemented, at a minimum. Staff must wear the following items:

- surgical mask

- fluid-resistant long-sleeved gown

- goggles or face shield

- gloves

5.5 Recommended PPE for staff caring for COVID-19-infected patients includes added precautions for patients with significant respiratory illness, when aerosol-generating procedures are likely and/or prolonged or very close contact with the patient is likely. In these cases, airborne precautions are followed, including:

- an N95/P2 mask

- fluid-resistant long-sleeved gown

- goggles or face shield

- gloves

5.6 In addition, the following can be considered:

- hair cover for aerosol-generating procedures

- shoes that are impermeable to liquids and can be wiped down

Recurrent use of shoe covers is not recommended, as repeated removal is likely to increase the risk of staff contamination.

5.7 PPE must remain in place and be worn correctly for the duration of exposure to potentially contaminated areas. PPE (particularly masks) should not be adjusted during patient care.

5.8 Use a step-by-step process for donning and doffing PPE as per local guidelines.

5.9 Changing into scrubs is recommended to staff and encouraged to get changed out of their uniform before leaving work and to transport worn uniforms home in a plastic bag for washing at home. 


\section{Continued}

5.10 Minimise personal effects in the workplace. All personal items should be removed before entering clinical areas and donning PPE. This includes earrings, watches, lanyards, mobile phones, pagers, pens, etc.

Stethoscope use should be minimised. If required, use dedicated stethoscopes within isolation areas.

Hair should be tied back out of the face and eyes.

5.11 Staff caring for infectious patients must apply correct PPE, irrespective of physical isolation. For example, in ICU, if patients are cohorted into a Pod with open rooms, staff working within the confines of the ICU Pod but not directly involved in patient care should also wear PPE. The same applies once infectious patients are nursed on an open ward. Staff then use plastic aprons, a change of gloves and hand hygiene when moving between patients in open areas.

5.12 When a unit is caring for a patient with confirmed or suspected COVID-19, it is recommended that all donning and doffing are supervised by an additional appropriately trained staff member.

5.13 Avoid sharing equipment. Preferably only use single-use equipment.

5.14 Wear an additional plastic apron if high volume of fluid exposure is expected.

5.15 If reusable PPE items are used (eg, goggles), these must be cleaned and disinfected prior to re-use.

\section{Conclusion}

Due to the peculiarity of COVID-19 as a highly communicable/infectious disease with attendant respiratory and other physical complications, training and utilization of specialized physiotherapy services has become very important for the purpose of providing effective and qualitative services. This position paper is expected to serve as the minimum requirement for participation of physiotherapists as frontline healthcare workers in the battle against COVID-19, with minimal or no risk to both staff and patients. Since training materials are highly essential to guide effective treatment procedures, our aim is to also compile the assessments and treatment guidelines available worldwide to guide Nigerian physiotherapists. These guidelines could be adjusted or modified to fit specific clinical settings across the nation.

\section{Conflicts of Interest}

The authors declare no conflicts of interest regarding the publication of this paper.

\section{References}

[1] Huang, C., Wang, Y., Li, X., Ren, L., Zhao, J., Hu, Y., et al. (2020) Clinical Features of Patients Infected with 2019 Novel Coronavirus in Wuhan, China. The Lancet, 395 497-506. https://doi.org/10.1016/S0140-6736(20)30183-5

[2] World Health Organization Novel Coronavirus (2019-nCoV). https://www.who.int/emergencies/diseases/novel-coronavirus-2019

[3] Adhikari, S.P., et al. (2020) Epidemiology, Causes, Clinical Manifestation and Diagnosis, Prevention and Control of Coronavirus Disease (COVID-19) during the Early Outbreak Period: A Scoping Review. BMC Infectious Disease of Poverty, 9, Article No. 29. https://doi.org/10.1186/s40249-020-00646-X

[4] European Centre for Disease Prevention and Control. https://www.ecdc.europa.eu

[5] Nigeria Centre for Disease Control (2020) Part of a Graphic Created for Disease Control. 
[6] https://www.hrw.org/news/2020/04/14/nigeria-protect-most-vulnerable-covid-19-re sponse

[7] https://www.theguardian.com/world/2020/apr/28/nigerian-authorities-deny-wave-o f-deaths-is-due-to-covid-19

[8] Wu, Y.-C., Chen, C.-S. and Chan, Y.-J. (2020) The Outbreak of COVID-19: An Overview. Journal of the Chinese Medical Association, 83, 217-220. https://doi.org/10.1097/JCMA.0000000000000270

[9] https://www.ersnet.org/covid-19-blog/covid-19-in-the-acute-setting-the-italian-ass ociation-of-respiratory-physiotherapists-position-paper

[10] Wujtewicz, M., Dylczyk-Sommer, A., Aszkiełowicz, A., et al. (2020) COVID-19 What Should Anaesthesiologists and Intensivists Know about It? Anaesthesiology Intensive Therapy, 52, 34-41. https://doi.org/10.5114/ait.2020.93756

[11] Kallet, R.H., Hemphill, J.C., Dicker, R.A., et al. (2007) The Spontaneous Breathing Pattern and Work of Breathing of Patients with Acute Respiratory Distress Syndrome and Acute Lung Injury. Respiratory Care, 52, 989-995.

[12] Lazzeri, M., Lanza, A., Bellini, R., Bellofiore, A., Cecchetto, S. and Colombo, A. (2020) Respiratory Physiotherapy in Patients with COVID-19 Infection in Acute Setting: A Position Paper of the Italian Association of Respiratory Physiotherapists. Monaldi Archives for Chest Disease, 90, 1285. https://doi.org/10.4081/monaldi.2020.1285

[13] Seto, W.H., Tsang, D., Yung, R.W., et al. (2003) Effectiveness of Precautions against Droplets and Contact in Prevention of Nosocomial Transmission of Severe Acute Respiratory Syndrome (SARS). The Lancet, 361, 1519-1520. https://doi.org/10.1016/S0140-6736(03)13168-6

[14] Barakzai, M.D. and Fraser, D. (2008) Assessment of Infection in Older Adults: Signs and Symptoms in Four Body Systems. Journal of Gerontological Nursing, 34, 7-12. https://doi.org/10.3928/00989134-20080101-02

[15] https://www.wcpt.org/news/Novel-Coronavirus-2019-nCoV

[16] Goroll, A.H. and Mulley, A.G. (2006) Primary Care Medicine: Office Evaluation and Management of the Adult. 5th Edition, Lippincott Williams \& Wilkins, Philadelphia, 314-329.

[17] Willis, L.M. (2007) Health Assessment Made Incredibly Visual! Lippincott Williams \& Wilkins, Philadelphia, 70-85.

[18] Sharma, G. and Goodwin, J. (2006) Effect of Aging on Respiratory System Physiology and Immunology. Clinical Interventions in Aging, 1, 253-260. https://doi.org/10.2147/ciia.2006.1.3.253

[19] Colleton, L.G. (2008) Beyond the Stethoscope: Respiratory Assessment of the Older Adult. Nursing Made Incredibly Easy, 6, 11-14. https://doi.org/10.1097/01.NME.0000334098.82242.98

[20] Arnold, F.W., et al. (2009) Improving Outcomes in Elderly Patients with Community-Acquired Pneumonia by Adhering to National Guidelines. Archives of Internal Medicine, 169, 1515-1524. https://doi.org/10.1001/archinternmed.2009.265

[21] Thomas, P., Baldwin, C., Bissett, B., Boden, I., Gosselink, R., Granger, C.L., Hodgson, C., Jones, A.Y., Kho, M.E., Moses, R., Ntoumenopoulos, G., Parry, S.M., Patman, S. and van der Lee, L. (2020) Physiotherapy Management for COVID-19 in the Acute Hospital Setting: Clinical Practice Recommendations. Journal of Physiotherapy, 66, 73-82. https://doi.org/10.1016/j.jphys.2020.03.011 